\title{
Pistes bibliographiques
}

\section{Christine Collière-Whiteside et Karine Meshoub-Manière}

\section{OpenEdition}

\section{Journals}

\section{Édition électronique}

URL : http://journals.openedition.org/genesis/4216

DOI : 10.4000/genesis.4216

ISSN : 2268-1590

\section{Éditeur :}

Presses universitaires de Paris Sorbonne (PUPS), Société internationale de génétique artistique littéraire et scientifique (SIGALES)

\section{Édition imprimée}

Date de publication : 1 juin 2019

Pagination : 155-156

ISSN : 1167-5101

\section{Référence électronique}

Christine Collière-Whiteside et Karine Meshoub-Manière, «Pistes bibliographiques », Genesis [En ligne], 48 | 2019, mis en ligne le 01 juin 2019, consulté le 25 janvier 2021. URL : http://

journals.openedition.org/genesis/4216; DOI : https://doi.org/10.4000/genesis.4216 


\section{Pistes bibliographiques}

\section{Fac-similés, témoignages, correspondances}

ALDRIDGE Jeffrey (dir.), The best of Bookmark: children's writers talk about their work, Edinburgh, Moray House Publications, 1994. Entretiens avec des auteurs britanniques : Leon Garfield, Anne Fine, Allan Baillie, Norman Maccaig, Joan Lingard, Gene Kemp, Robert Cormier, Farrukh Dhondy, Terrance Dicks, Tomie de Paola, Eric Carle, Raymond Briggs, Bernard Ashley, Judith O’Neill, Iona McGregor, Rosemary Sutcliff.

ANDRIEU Brigitte et LoRANT-Jolly Annick, «Entretien avec François Place», La Revue des livres pour enfants, $\mathrm{n}^{\circ} 254,2010$, p. 101-112.

Antoine-Andersen, Véronique, Conversation avec Kitty Crowther, Paris, Pyramid Éditions, 2016.

Beckett Sandra L., De grands romanciers écrivent pour les enfants, Montréal, PU de Montréal, 1997.

Blake Quentin, La Vie de la page, Paris, Gallimard, 1995.

Blake Quentin, Words and Pictures, London, Jonathan Cape, 2000 [traduction française : Pages, mots, images, Paris, Gallimard, 2014].

CARroll Lewis, Alice's Adventures under Ground: Turning the Pages of Lewis Carroll's Original Manuscript, British Library, 2005, également consultable sur la galerie en ligne de la British Library : http://www. bl.uk/onlinegallery/ttp/ttpbooks.html

Cerisier Alban et Desse Jacques, De la jeunesse chez Gallimard : 90 ans de livres pour enfants. Un catalogue, Paris, Gallimard chez les Libraires Associés, 2008.

Cohen Morton N. et Wakeling Edward (dir.), Lewis Carroll and His Illustrators : Collaborations and Correspondence, 1865-1898, London, Macmillan, 2003.

Cohen Morton N. et Gandolfo Anita, Lewis Carroll and the House of Macmillan, Cambridge, Cambridge UP, 1987.

DuhÊme Jacqueline, «L'Opéra de la lune», dans Bachelot-Prévert E. et Binh N. T., Jacques Prévert, Paris la belle, Paris, Flammarion, 2008, p. 132-141.

Duras Marguerite et Couprie Katy (ill.), Ah! Ernesto, Paris, Thierry Magnier, 2013.

Duras Marguerite et Couprie Katy (ill.), Ah! Duras, Paris, Thierry Magnier, 2013.

Elzbieta, L'Enfance de l'art, Rodez, Le Rouergue, 1997.

SAInT-Exupéry, Antoine de, Le manuscrit du «Petit Prince» d'Antoine de Saint-Exupéry : fac-similé et transcription, Alban Cerisier et Delphine Lacroix (dir.), Paris, Gallimard, 2013.
Pef, Enfants de papier, Paris, Gallimard, 1993

Pommaux Yvan, Yvan Pommaux : correspondance entre Yvan Pommaux et Lucie Cauwe, Paris, l'école des loisirs, coll. «Tout sur votre auteur préféré », 2014.

Pommaux Yvan, Yvan Pommaux au travail : La Commune, dans la collection des Grandes images de l'Histoire, documentaire portant sur la genèse de l'album La Commune, des premiers avant-textes à l'impression finale, https://www.ecoledesloisirs.fr/auteur/yvan-pommaux.

ROGINSKI Jim, Behind the covers: interviews with authors and illustrators of books for children and young adults, vol. I, Littleton (Colo.), Libraries unlimited 1985, et vol. II, Englewood (Colo.), Libraries unlimited, 1989.

VAn der Linden Sophie, Claude Ponti, Paris, Éditions Être, coll. «Boîtazoutils», 2000.

Voltz Christian, Dans l'atelier de Christian Voltz, Rodez, Le Rouergue, 2011. Cahier d'activités destiné à des élèves de primaire, où ils sont invités à imiter les techniques de l'auteur, à partir d'exemples tirés de son atelier.

\section{Études génétiques}

\section{Ouvrages}

Genesis, $\mathrm{n}^{\circ} 43$ «Bande Dessinée», Pierre-Marc de Biasi et Luc Vigier (dir.), Paris, SUP, 2016.

Bon François, François Place Illustrateur, Paris, Casterman, 1994.

Boucherville Adèle de, La Fabrique d'Olivier Douzou, Le Puy-en-Velay, L'atelier du poisson soluble, coll. «La fabrique», 2015.

Boucherville Adèle de, La Fabrique de Claude Ponti, Le Puy-en-Velay, L'atelier du poisson soluble, coll. «La fabrique», 2016.

Boucherville Adèle de, La Fabrique de Bruno Heitz, Le Puy-en-Velay, L'atelier du poisson soluble, coll. «La fabrique», à paraître.

Brunhoff Jean de, Brunhoff Laurent de et Fournel Paul, Babar, impressions, Paris, Hachette jeunesse, 2006.

Butcher William, Jules Verne inédit : les manuscrits déchiffrés, Lyon, ENS éditions : Institut d'histoire du livre, 2015.

Charles Dorothée (dir.), Les Histoires de Babar, Paris, les Arts décoratifs : BnF, 2011, catalogue de l'exposition «La fabrique de Babar».

Clermont Philippe et Henky Danièle (dir.), Littérature de jeunesse : la fabrique de la fiction, Francfort-sur-le-Main, Peter Lang, 2017.

Delbard Claire, Le Père Castor en poche (1980-1990) ou comment innover sans trahir?, Paris, L'Harmattan, 2007. 
Letourneux Matthieu et Mollier Jean-Yves, La librairie Tallandier : histoire d'une grande maison d'édition populaire (1870-2000), Paris, Nouveau Monde Éditions, 2011.

Mollier Jean-Yves, Louis Hachette (1800-1864) : le fondateur d'un empire, Paris, Fayard, 1999.

Nelson Christine, Drawing Babar: Early drafts and watercolours, New York, The Morgan Library and Museum, 2008.

Nières-Chevrel Isabelle, Au pays de Babar : les albums de Jean de Brunhoff, Rennes, PUR, 2017.

Perrot Jean, Carnets d'illustrateurs, Éditions du Cercle de la librairie, 2000. 236 p. ill.; $22 \mathrm{~cm}+1$ CDrom PC/Mac.

PIffault Olivier (dir.), Babar, Harry Potter \& Cie : Livres d'enfants d'hier et d'aujourd'hui, Paris, BnF, 2008.

PLu Christine, Georges Lemoine : illustrer la littérature au $\mathrm{xx}^{e}$ siècle, thèse de doctorat soutenue en 2005 à l'université Rennes 2, sous la direction d'Isabelle Nières-Chevrel.

\section{Articles}

BAZIN Laurent, «De l'émergence des images : ontogenèse de la création chez François Place», dans Clermont Ph. et Henky D. (dir.), Littérature de jeunesse : la fabrique de la fiction, Francfort-sur-le-Main, Peter Lang, 2017, p. 131-142.

BEDoIn Evelyne, «La collection de jouets Tomi Ungerer, une archive de 1'œuvre?», dans Clermont Ph. et Henky D. (dir.), Littérature de jeunesse : la fabrique de la fiction, Francfort-sur-le-Main, Peter Lang, 2017, p. 61-78.

BURAT Juliette, «De la rencontre avec un auteur de littérature jeunesse à la production d'écrit littéraire en cycle 3 », dans Jacques M. et RauletMarcel C. (dir.), Inventions de l'écriture, Dijon, EUD, 2014, p. 213-229.

Boucheron Sabine, «Discours des origines et traces discursives : histoire d'une rature légendaire », à propos du poème «Liberté » de Paul Éluard, dans Langage et société, 2001/3, n 97, p. 71-97.

Boucherville Adèle de, «La petite fabrique de Bruno Heitz», La Revue des livres pour enfants, section «Libre Parcours », septembre 2014, $\mathrm{n}^{\circ} 278$, p. 149-161.

CAMENish Annie, «Du mythe américain au jeu langagier dans Il était une oie dans l'Ouest de Damien Chavanat», dans Clermont Ph. et Henky D. (dir.), Littérature de jeunesse : la fabrique de la fiction, Francfortsur-le-Main, Peter Lang, 2017, p. 161-176.

Ospina Canencio Cielo Erika et Macuada July, «De canción de cuna a narración ilustrada : una aproximación crítico-genética al libro para niños Duerme, niño duerme », Manuscrítica. Revista de Crítica Genética, $\mathrm{n}^{\circ} 33,2017$, p. 90-108.

Clermont Philippe, «Du Cour d'Écogée à Écoland, éléments de la genèse d'un roman de Christian Grenier», dans Clermont Ph. et Henky D. (dir.), Littérature de jeunesse : la fabrique de la fiction, Francfort-sur-le-Main, Peter Lang, 2017, p. 177-192.

COLliÈre-Whiteside Christine, «Illustrating the Sylvie and Bruno books: the collaboration of Lewis Carroll and Harry Furniss », Recto/Verso, $\mathrm{n}^{\circ} 3$, juin 2008, en ligne.
CoJEz Anne-Marie, «Le dossier préparatoire de En famille», dans Hector Malot et le métier d'écrivain, Marcoin F. (dir.), Paris, Magellan \& Cie, 2008, p. 85-99.

CoJez Anne-Marie, «Plans et documents pour En famille : une approche génétique du roman d'Hector Malot», L'Euvre pour la jeunesse d'Hector Malot (une lecture contemporaine internationale), Paris, L'Harmattan, 2009, p. 19-37.

FranÇOIS Cyrille, «Andersen trouve-t-il son conte? De "Dødningen" ("Le mort", 1830) à "Reisekammeraten" ("Le compagnon de voyage", $1835)$ ), Genesis, n $^{\circ} 44$, «Après le texte. De la réécriture après publication», 2017, p. 65-79.

Grenier Christian, «Les archives de mes textes », dans Clermont Ph. et Henky D. (dir.), Littérature de jeunesse : la fabrique de la fiction, Francfort-sur-le-Main, Peter Lang, 2017, p. 93-115.

Henky Danièle, «L'homme qui plantait des arbres de Giono : histoire de la mystification d'une genèse de texte», dans Clermont $\mathrm{Ph}$. et Henky D. (dir.), Littérature de jeunesse : la fabrique de la fiction, Francfort-sur-le-Main, Peter Lang, 2017, p. 193-203.

LABADENS François, «Le manuscrit de Diloy le Chemineau», Les Cahiers Séguriens, $\mathrm{n}^{\circ} 6$ «Diloy le Chemineau», Association des Amis de la Comtesse de Ségur, 2006, p. 75-80.

PosLANIEC Christian, «Une étape alchimique dans la création d'un livre», dans Clermont $\mathrm{Ph}$. et Henky D. (dir.), Littérature de jeunesse : la fabrique de la fiction, Francfort-sur-le-Main, Peter Lang, 2017, p. 79-91.

RECTOR Gretchen V., «Digging in the Garden: the Manuscript of The Secret Garden by Frances Hodgson Burnett», dans The Secret Garden, Norton Critical Editions, Gretchen Holbrook Gerzina (dir.), Norton, 2006, p. 186-199.

SAUdray Rémi, «L'art de conclure», Les Cahiers Séguriens, n² «Le Général Dourakine», Association des Amis de la Comtesse de Ségur, 2001, p. 64-71.

SaUdray Rémi, «Le découpage en chapitres de Diloy le Chemineau», Les Cahiers Séguriens, n 6 «Diloy le Chemineau», Association des Amis de la Comtesse de Ségur, 2006, p. 81-89.

SAUDRAY Rémi et LABADENS François, «Et pourquoi pas une trilogie?», Les Cahiers Séguriens, $n^{\circ} 9$ «La Trilogie», Association des Amis de la Comtesse de Ségur, 2010, p. 13-26.

STRICH Marie-José, «Critique génétique d'un manuscrit : La Bible d'une grand-mère (1869)», Cahiers Robinson, $\mathrm{n}^{\circ}$ 9, La Comtesse de Ségur et ses alentours, 2001, p. 183-192.

SCheinhardt Philippe, «Jules Verne : un processus d'écriture sous contraintes », Genesis, n 33, 2011, p. 173-186.

ScheinhardT Philippe, «Jules Verne sous la tutelle d'Hetzel. La question de la censure dans le manuscrit d'Une ville flottante», dans Viollet C. et Bustarret C. (dir.), Genèse, censure, autocensure, Paris, CNRS Éditions, 2005.

VERGEZ-SANS Cécile, «Les carnets d'Olivier Douzou, création et travail d'édition », dans Clermont Ph. et Henky D. (dir.), Littérature de jeunesse : la fabrique de la fiction, Francfort-sur-le-Main, Peter Lang, 2017, p. 143-159. 